PROCEEDINGS OF THE

AMERICAN MATHEMATICAL SOCIETY

Volume 105, Number 4, April 1989

\title{
A REMARK ON RADON-NIKODYM PROPERTIES OF ORDERED HILBERT SPACES
}

\author{
LOTHAR M. SCHMITT
}

(Communicated by Paul S. Muhly)

\begin{abstract}
We characterize classical $L^{2}$-spaces as well as $L^{2}$-spaces of $W^{*}$ algebras by appropriate Radon- Nikodym principles.
\end{abstract}

Segal [9] has shown that usual measure theory can be extended reasonably only to measure spaces, whose corresponding $L^{1}$-spaces have the RadonNikodym property. The aim of this note is to show that $L^{2}$-spaces obtained via commutative, respectively, noncommutative integration are as well determined as ordered spaces by suitable Radon-Nikodym principles. Order theoretical characterizations of classical $L^{2}$-spaces were obtained by Sz.-Nagy [11] in the separable and Schaefer [5] in the general case. Noncommutative $L^{2}$ spaces were characterized by Connes [2] and Wittstock and the author [8, 6]. Radon-Nikodym properties of these $L^{2}$-spaces were established by Connes [2], Stratila-Zsido [10] and in [7].

Suppose $\mathscr{H}$ is a Hilbert space, which is ordered by a self-dual cone $\mathscr{H}^{+}$. Let $J$ be the antilinear unitary involution associated with $\mathscr{H}^{+}$by [2, Proposition 4.1]. Furthermore let $\mathscr{M}_{h}$ be the ideal center of $\left(\mathscr{H}, \mathscr{H}^{+}\right)$in the sense of Wils-see [1, p. 76] -and $\mathscr{M}=\mathscr{M}_{h} \oplus i \mathscr{M}_{h}$. Bös [1] has shown that $\mathscr{M}$ is a commutative $W^{*}$-algebra with involution $x^{*}=J x J, x \in \mathscr{M}$. In particular the cones of operator-positive, respectively, order-positive elements in $\mathscr{M}$ coincide.

Definition. $\left(\mathscr{H}, \mathscr{H}^{+}\right)$has the Radon-Nikodym property if for $\eta, \xi \in \mathscr{H}^{+}$ satisfying $\eta \leq \xi$ there exists $x \in \mathscr{M}^{+}$such that $\eta=x \xi$.

Proposition 1. $\left(\mathscr{H}, \mathscr{H}^{+}\right)$has the Radon-Nikodym property if and only if $\left(\mathscr{H}, \mathscr{H}^{+}\right)$is unitarily equivalent to $\left(L^{2}(X, \mu), L^{2}(X, \mu)^{+}\right)$for a measure space $(X, \mu)$ in the sense of Segal [9].

We shall give the proof of Proposition 1 in a moment. If $V$ is a linear space, then we shall write $V_{n}=M_{n}(V)$ for the space of $n \times n$ matrices with entries in $V$. Again let $\mathscr{H}$ be a Hilbert space and suppose that $\left\{\mathscr{H}_{n}^{+}, n \in \mathbf{N}\right\}$ is a

Received by the editors October 19, 1987 and, in revised form, August 30, 1988.

1980 Mathematics Subject Classification (1985 Revision). Primary 46L50, 46B30.

Supported in part by NSF grant \# DMS 8403631 a.4. 
family of self-dual cones $\mathscr{H}_{n}^{+} \subseteq \mathscr{H}_{n}$ such that $\left(\mathscr{H}, \mathscr{H}_{n}^{+}\right)$is a matrix ordered space. Let $J_{n}$ be the involution in $\mathscr{H}_{n}$ associated with $\mathscr{H}_{n}^{+}$. Finally let $\mathscr{M}$ be the matrix-multiplier algebra of $\left(\mathscr{H}, \mathscr{H}_{n}^{+}, n \in \mathbf{N}\right)$ in the sense of [8, Definition 2.1].

Definition. $\left(\mathscr{H}, \mathscr{H}_{n}^{+}, n \in \mathbf{N}\right)$ has the matricial Radon-Nikodym property if for $n \in \mathbf{N}, \xi, \eta \in \mathscr{H}_{n}^{+}$satisfying $\eta \leq \xi$ there exists $x \in \mathscr{M}_{n}^{+}$such that $\eta=x J_{n} x J_{n} \xi$.

Proposition 2. ( $\mathscr{H}, \mathscr{H}_{n}^{+}, n \in \mathbf{N}$ ) has the matricial Radon-Nikodym property if and only if $\left(\mathscr{M}, \mathscr{H}^{n}, \mathscr{H}_{n}^{+}, n \in \mathbf{N}\right)$ is a matrix-ordered standard form in the sense of [8, Definition 1.4].

Proof of Proposition 1. Suppose $\left(\mathscr{H}, \mathscr{H}^{+}\right)$has the Radon-Nikodym property. Let $\xi_{\alpha}, \eta_{\alpha} \in \mathscr{H}^{+} ; \alpha, \beta=1,2$ and $\xi=\xi_{1}+\xi_{2}=\eta_{1}+\eta_{2}$. Then $\xi_{\alpha}=x_{\alpha} \xi$, $\eta_{\beta}=y_{\beta} \xi$ for $x_{\alpha}, y_{\alpha} \in \mathscr{M}^{+}$. Let $\rho_{\alpha \beta}=x_{\alpha} y_{\beta} \xi$. Then $\xi_{\alpha}=\sum_{\beta} \rho_{\alpha \beta}$ and $\eta_{\beta}=\sum_{\alpha x} \rho_{\alpha \beta}$. Hence $\left(\mathscr{H}, \mathscr{H}^{+}\right)$has the Riesz decomposition property and therefore satisfies the hypothesis of [6, Corollary 2.6]. There is one and only one way to define a matrix order on $\mathscr{H}$ with by uniqueness self-dual cones $\mathscr{H}_{n}^{+} \subseteq \mathscr{H}_{n}$ such that $\mathscr{H}_{1}^{+}=\mathscr{H}^{+} .\left(\mathscr{H}, \mathscr{H}_{n}^{+}, n \in \mathbf{N}\right)$ satisfies the hypothesis (IV) of [6, Theorem 3.2]. Let $\mathscr{N}$ be the matrix multiplier algebra of $\left(\mathscr{H}, \mathscr{H}_{n}^{+}\right)$. Since the cones $\mathscr{H}_{n}^{+}$coincide with their transpose

$$
\mathscr{H}_{n}^{+\mathrm{T}}=\left\{\left(\xi_{i j}\right) \mid\left(\xi_{j i}\right) \in \mathscr{H}_{n}^{+}\right\}
$$

and $\left(\mathscr{N}^{\prime}, \mathscr{H}, \mathscr{H}_{n}^{+\mathrm{T}}\right)$ is a matrix ordered standard form we have $\mathscr{N}=\mathscr{N}^{\prime}$. Hence $\mathscr{N}=\mathscr{M}$ is a commutative $W^{*}$-algebra in standard form. Applying [3, Theorem 2.3] and [4, Proposition 1.18.1] completes the proof.

Note that the above argument can also be used to show that a Hilbert lattice is order isomorphic to an $L^{2}$-space.

Proof of Proposition 2. Suppose $\left(\mathscr{H}, \mathscr{H}_{n}^{+}, n \in \mathbf{N}\right)$ has the matricial RadonNikodym property. Let $n \in \mathbf{N}$ be fixed, $\xi, \eta \in \mathbf{H}_{n}^{+}$such that $\xi \perp \eta$. Then $\xi=x J_{n} x J_{n}(\xi+\eta)$ for some $x \in \mathscr{M}_{n}^{+}$. Now we have

$$
\left\langle\left(\begin{array}{cc}
x J_{n} x J_{n} \eta & x \eta \\
J_{n} x J_{n} \eta & \eta
\end{array}\right), \quad \eta \otimes \gamma\right\rangle \geq 0 \quad \text { for } \gamma \in \mathscr{M}_{2}(\mathbf{C})^{+} .
$$

Hence

$$
\left(\begin{array}{cc}
0 & \langle x \eta, \eta\rangle \\
\left\langle J_{n} x J_{n} \eta\right\rangle & \langle\eta, \eta\rangle
\end{array}\right)
$$

is a positive matrix and $\langle x \eta, \eta\rangle=0=\langle p \eta, \eta\rangle$ where $p=\operatorname{supp}(x) \in \mathscr{M}$ by [8, Theorem 2.2]. Hence we have $p \eta=J_{n} p J_{n} \eta=0$ and $\xi=p \xi=J_{n} p J_{n} \xi$. Now one can prove $[8$, Lemma 4.1 ; Theorem 4.3 (ii) $\Rightarrow$ (i)] under the above hypothesis. The converse direction in the proof of Proposition 2 is established in [7]. 


\section{REFERENCES}

1. W. Bös, A classification for selfdual cones in Hilbert spaces, Arch. Math. (Basel) 30 (1978), 75-82.

2. A. Connes, Charactérisation des éspaces vectoriels ordonnés sans jacents aux algèbres de von Neumann, Ann. Inst. Fourier (Grenoble) 24 (1974), 121-155.

3. U. Haagerup, The standard form of von Neumann algebras, Math. Scand. 37 (1975), 271-283.

4. S. Sakai, $C^{*}$-algebras and $W^{*}$-algebras, Ergebnisse der Mathematik und ihrer Grenzgebiete, Bd. 60, Spinger-Verlag, Berlin, 1971.

5. H. H. Schaefer, Banach lattices and positive operators, Grundlehren Math. Wiss., Bd. 215, Springer-Verlag, Berlin, 1974.

6. L. M. Schmitt, Characterization of $L^{2}(\mathscr{M})$ for injective $W^{*}$-algebras $\mathscr{M}$, Math. Scand. 57 (1985), 267-280.

7. _ The Radon-Nikodym theorem for $L^{p}$-spaces of $W^{*}$-algebras, Publ. Res. Inst. Math. Sci. 22 (1986), 1025-1034.

8. L. M. Schmitt and G. Wittstock, Characterization of matrix ordered standard forms of $W^{*}$ algebras, Math. Scand. 51 (1982), 241-260.

9. J. E. Segal, Equivalence of measure spaces, Amer. J. Math. 73 (1951), 275-313.

10. S. Stratila and L. Zsido, Lectures on von Neumann algebras, Abacus Press, Tunbridge Wells, England, 1979.

11. B. D. Sz.-Nagy, On the set of positive functions in $L_{2}$, Ann. of Math. II. 39 (1938), 1-13.

Universitaet Osnabrueck, Fachbereich Mathematik/Informatik, D-4500 Osnabrueck, West Germany 